\title{
Numerically Converged Solutions of the Global Primitive Equations for Testing the Dynamical Core of Atmospheric GCMs
}

\author{
L. M. Polvani \\ Department of Applied Physics and Applied Mathematics, and Department of Earth and Environmental Sciences, Columbia University, \\ New York, New York \\ R. K. SсотT \\ Department of Applied Physics and Applied Mathematics, Columbia University, New York, New York
}

S. J. THOMAS

Scientific Computing Division, National Center for Atmospheric Research, Boulder, Colorado

(Manuscript received 5 October 2002, in final form 22 March 2004)

\begin{abstract}
Solutions of the dry, adiabatic, primitive equations are computed, for the first time, to numerical convergence. These solutions consist of the short-time evolution of a slightly perturbed, baroclinically unstable, midlatitude jet, initially similar to the archetypal LC1 case of Thorncroft et al. The solutions are computed with two distinct numerical schemes to demonstrate that they are not dependent on the method used to obtain them.

These solutions are used to propose a new test case for dynamical cores of atmospheric general circulation models. Instantaneous horizontal and vertical cross sections of vorticity and vertical velocity after 12 days, together with tables of key diagnostic quantities derived from the new solutions, are offered as reproducible benchmarks. Unlike the Held and Suarez benchmark, the partial differential equations and the initial conditions are here completely specified, and the new test case requires only 12 days of integration, involves no spatial or temporal averaging, and does not call for physical parameterizations to be added to the dynamical core itself.
\end{abstract}

\section{Introduction}

The development of atmospheric general circulation models (AGCMs) is an important task among the current efforts to understand and predict the climate. At the heart of every AGCM is the so-called dynamical core, the component of the model that deals with the numerical solution of the dry, adiabatic, primitive equations. While in many ways a much simpler task than the design of other model components, the search for ever more efficient numerical solvers for the primitive equations is an important area of research, often necessitated by the continual evolution of computer architectures.

In order to build new reliable dynamical cores and quantitatively evaluate their accuracy, it is important to test their solutions against known ones. The first step in this direction was taken by Williamson et al. (1992,

Corresponding author address: L. M. Polvani, Department of Applied Physics and Applied Mathematics, Columbia University, 500 West 120th St., Arm. 216, New York, NY 10027.

E-mail: polvani@columbia.edu hereafter W92), who proposed a set of "standard tests" for the shallow-water equations in spherical geometry. While useful in their own right, the tests in the W92 set present three major limitations. First, they cannot be used to validate AGCM dynamical cores directly, since the shallow-water equations lack the dependence on a vertical coordinate. Second, most of the flows in the W92 tests are unrealistically simple compared to the kind of flows that need to be computed with typical AGCMs. Third, and most importantly, in recent years it has been shown that the most dynamically interesting tests in W92 (tests 5 and 6) are in fact very difficult to compute accurately; this severely limits their practical utility. For a more complete discussion, see Galewsky et al. (2004).

A second important step in the development of test cases for dynamical cores was the proposal of Held and Suarez (1994, hereafter HS94). They suggested adding analytically specified parameterizations of two simple physical processes (Newtonian relaxation and surface drag) to a dynamical core, and then comparing the timemean, zonally averaged wind and temperature fields that result from a 1000-day integration. The averaging over 
such a long integration period is dictated by the desire to evaluate the long-term statistics of the computed circulation, an important objective for any AGCM. Although extremely idealized, the HS94 system is capable of reproducing key features of the tropospheric circulation and hence provides a realistic benchmark for the comparison of dynamical cores.

One drawback of the HS94 test case is that the quantities to be compared involve both temporal and spatial averaging. One important application of dynamical core test cases is the debugging of new cores, and it is easy to imagine how subtle programming errors might be masked by the temporal and spatial averaging. Another drawback of the HS94 test case is that the authors did not show actual numerical convergence of their solutions, although this issue has been partially addressed by Boer and Dennis (1997). A final minor drawback of the HS94 test case is the fact that new physical parameterizations need to be added to the dynamical core itself; ideally, one would want to test an AGCM dynamical core by simply switching off all physics with a flag, and then integrating the dry, adiabatic, primitive equations from a given initial condition, without the need to add more code.

In order to address these shortcomings, we here propose a new test case that involves a short integration of the unforced primitive equations, and we accurately compute the solutions to be used as benchmarks. Unlike the HS94 test case, the new test case is a simple initial value problem and consists of solving a precisely specified set of equations (the primitive equations), with an analytically specified initial condition (a baroclinically unstable midlatitude jet), for a period of 12 days following a small initial perturbation. In order to construct benchmark solutions, we compute the evolution of the flow to numerical convergence. To the best of our knowledge, this is the first set of published solutions of the time-dependent, atmospheric, primitive equations in spherical coordinates for which numerical convergence has been attempted or achieved. Furthermore, we compute solutions with two completely distinct numerical schemes, thereby demonstrating that the solution to the new test case is model independent and should thus be reproducible with any future scheme. We provide both snapshots of the actual fields, plots of key norms, and tables of a few diagnostic quantities as references against which solutions from future dynamical cores may be contrasted.

From this it should be clear that the philosophy of the new test case presented here is fundamentally different from the one originally proposed by HS94 and common to most "model intercomparison" tests. In those, the goal is to set up different models so as to compute similar (if not identical) physical evolutions, and then compare the output of the different models with one another. For such tests, no unique or exact solution is known or believed to exist, and the exercise consists in contrasting the features produced by the dif- ferent models, presumably in an effort to determine which ones "do better" than the others.

However, for the development, debugging, and testing of dynamical cores, we suggest that the emphasis be placed on the mathematics and not the physics. To the degree that an identical set of partial differential equations is agreed upon (e.g., the primitive equations), and that identical initial and boundary conditions are used, any consistent numerical scheme ought to produce the same numerically converged solution. This is the purpose of the present paper: to provide one such solution to assist in the testing of future dynamical cores. We view this test as complementary to the HS94 benchmark. In practice, we envisage our new test case being performed first, to ensure that a newly developed core is able to reproduce known solutions to within agreed tolerances over short-time integration periods; once this test is successfully passed, an HS94 integration can be performed to ascertain the long-time statistics of the model.

The paper is organized as follows. In the next section we carefully describe the differential equations and the initial conditions to be used for the new test case. In section 3 we present the evolution of the flow over a 12-day period of integration, computed with a familiar pseudospectral model, and demonstrate how numerical convergence is achieved. In section 4 , we compute solutions to the same problem with a different numerical model, based on the spectral-element method, and show that the numerically converged solutions thus obtained are identical to those obtained with the pseudospectral model (up to a certain number of digits). In section 5, we discuss the effect of hyperdiffusion on the solutions, stressing the fact that identical differential equations must be integrated if one is attempting to reproduce the solutions presented here. We conclude with a summary of how our solutions can be used as a new test case, followed by a brief discussion.

\section{Test case specifications}

In this section we describe in detail the differential equations and initial conditions to be used for the new test case. It is important to note that, unlike the HS94 test case, we are aiming for complete reproducibility here. Hence, we stress that the specifications in this section need to be followed exactly if one is attempting to reproduce the solutions presented in the next section.

\section{a. The model equations}

In order for this test to be of practical applicability to the greatest number of atmospheric models, the differential equations to be integrated were chosen to be the dry, adiabatic, primitive equations in spherical coordinates, as these are at the heart of the vast majority of present-day AGCMs. For simplicity, we confine our attention here to the case in which $\sigma$ is used as the 
vertical coordinate. We recognize that the majority of comprehensive AGCMs use a hybrid vertical coordinate (i.e., one involving both $\sigma$ and pressure); in that case, setting the pressure coefficients to zero would be an easy way of reproducing the solutions presented below.

Although these differential equations are well known, we reproduce them here for the sake of clarity, reproducibility, and completeness. The horizontal velocity field $\mathbf{u}=(u, v)$, the temperature $T$, and the surface pressure $p_{s}$, satisfy the following four prognostic equations:

$$
\begin{aligned}
\frac{d \mathbf{u}}{d t}+f \mathbf{k} \times \mathbf{u}+\nabla \Phi+\frac{R T}{p_{s}} \nabla p_{s} & =\nu \nabla^{2} \mathbf{u} \\
\frac{d T}{d t}-\frac{\kappa T}{\sigma p_{s}} \omega & =\nu \nabla^{2} T \\
\frac{\partial p}{\partial t}+\int_{0}^{1} \nabla \cdot\left(p_{s} \mathbf{u}\right) d \sigma & =0,
\end{aligned}
$$

where $\boldsymbol{\nabla}$ contains only the horizontal derivatives, $\sigma \equiv$ $p / p_{s}$, the intermediate variables $\Phi$ and $\omega$ (the geopotential and pressure vertical velocity) are computed diagnostically from the definitions

$$
\begin{aligned}
& \Phi=-R \int_{1}^{\sigma} T d\left(\ln \sigma^{\prime}\right) \text { and } \\
& \omega=\sigma \mathbf{u} \cdot \nabla p_{s}-\int_{0}^{\sigma} \nabla \cdot\left(p_{s} \mathbf{u}\right) d \sigma^{\prime},
\end{aligned}
$$

and the material derivative is defined by

$$
\frac{d}{d t}=\frac{\partial}{\partial t}+\mathbf{u} \cdot \boldsymbol{\nabla}+\dot{\sigma} \frac{\partial}{\partial \sigma} .
$$

Our notation is completely standard and, for further details, the reader is referred to Durran (1999), where these expressions are derived.

For this set of equations, the appropriate boundary conditions are $\dot{\sigma}=0$ at $\sigma=1$ (the ground) and at $\sigma$ $=0$ (the "top" of the atmosphere). Note that we have explicitly included diffusive terms on the right-hand side of the momentum and temperature equations in (1). An explanation for the need to include these terms is given in section $2 \mathrm{c}$ below.

\section{b. The initial conditions}

In order to meaningfully test an atmospheric dynamical core, it seems reasonable to require that it be able to compute the short-time evolution of a nontrivial initial condition, that is, one that represents a physical event of importance for the atmospheric circulation. We could think of no more obvious choice than the formation of synoptic-scale eddies via baroclinic instability. As such eddies are well known to play an essential role in the circulation of the atmosphere, it would seem

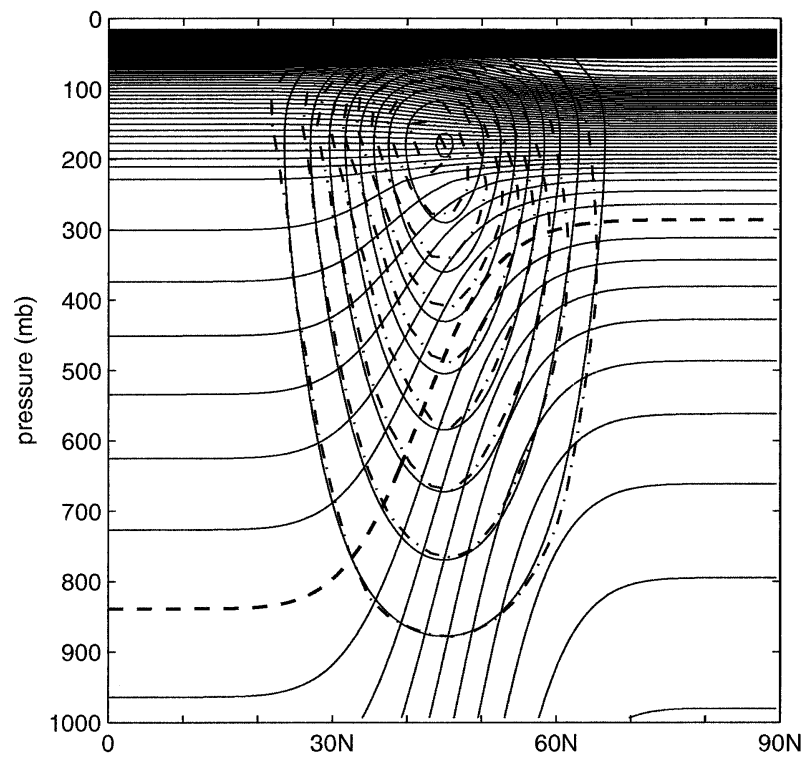

FIG. 1. The initial zonal wind from (5) with contour intervals of $5 \mathrm{~m} \mathrm{~s}^{-1}$ and the balanced potential temperature from (10) with contour intervals of $5 \mathrm{~K}$ (the dashed line is the $300-\mathrm{K}$ surface). The dashdotted lines are the zonal winds from Thorncroft et al. (1993).

that any decent dynamical core ought to be able to accurately compute their development.

The initial conditions for the new test case thus consist of a simple zonal flow, representing a typical midlatitude tropospheric jet, to which a small perturbation is added to induce the development of baroclinic instability. Both the basic flow and the initial perturbation are analytically specified, allowing the complete initial condition to be reproducible in testing future dynamical cores.

The basic flow, illustrated in Fig. 1, is constructed to be similar to the one used in the study of Thorncroft et al. (1993). This is easily accomplished by letting the zonal velocity $u$ be a simple product of a function of latitude $(\phi)$ and a function of pressure $(p)$

$$
u(\phi, p)= \begin{cases}u_{0} \sin ^{3}\left(\pi \mu^{2}\right) F(z) & \text { for } \phi>0 \\ 0 & \text { for } \phi<0\end{cases}
$$

where $\mu \equiv \sin \phi, z \equiv-H \log \left(p / p_{0}\right)$, and the vertical structure $F(z)$ is taken to be of the form

$$
F(z)=\frac{1}{2}\left[1-\tanh ^{3}\left(\frac{z-z_{0}}{\Delta z_{0}}\right)\right] \sin \left(\frac{\pi z}{z_{1}}\right) .
$$

With the numerical values of the parameters $u_{0}, z_{0}, \Delta z_{0}$, $z_{1}, H$, and $p_{0}$ given in Table 1 , the above expressions reproduce a zonal wind field that, below $200 \mathrm{hPa}$, is nearly identical to the one labeled LC1 in Thorncroft et al. (1993), as can be seen in Fig. 1. Of course, we set the meridional velocity $v=0$.

The temperature $T$ is initialized by combining the meridional momentum equation, 
TABLE 1. Numerical values of the parameters to be used for the new test case.

\begin{tabular}{lcl}
\hline \hline Parameter & Value & \multicolumn{1}{c}{ Units } \\
\hline$g$ & 9.806 & $\mathrm{~m} \mathrm{~s}^{-2}$ \\
$a$ & $6.371 \times 10^{6}$ & $\mathrm{~m}$ \\
$\Omega$ & $7.292 \times 10^{-5}$ & $1 \mathrm{~s}^{-1}$ \\
$R$ & 287 & $\mathrm{~J} \mathrm{~kg}^{-1} \mathrm{~K}^{-1}$ \\
$\kappa$ & $2 / 7$ & \\
$c_{p}$ & $R / \kappa$ & $\mathrm{J} \mathrm{kg} \mathrm{K}^{-1}$ \\
$p_{0}$ & $10^{5}$ & $\mathrm{~Pa}$ \\
$H$ & 7.34 & $\mathrm{~km}^{-1}$ \\
$u_{0}$ & 50 & $\mathrm{~m} \mathrm{~s}$ \\
$z_{0}$ & 22 & $\mathrm{~km} \mathrm{~km}^{-1}$ \\
$\Delta z_{0}$ & 5 & $\mathrm{~km}^{-1}$ \\
$z_{1}$ & 30 & $\mathrm{Kadians}$ \\
$\hat{T}$ & 1 & $\mathrm{radians}$ \\
$\lambda_{0}$ & 0 & \\
$\phi_{0}$ & $\pi / 4$ & \\
$\alpha$ & $1 / 3$ & $\mathrm{~m} \mathrm{~s}^{-1}$ \\
$\beta$ & $1 / 6$ & \\
$\nu$ & $7.0 \times 10^{5}$ &
\end{tabular}

$$
(a f+u \tan \phi) u=-\frac{\partial \Phi}{\partial \phi},
$$

and the hydrostatic balance relation (equivalent to 2),

$$
\frac{\partial \Phi}{\partial z}=\frac{R}{H} T
$$

to obtain

$$
\frac{\partial T}{\partial \phi}=-H R^{-1}(a f+2 u \tan \phi) \frac{\partial u}{\partial z},
$$

where $f=2 \Omega \sin \phi$. Again, the precise numerical values of $R, a$, and $\Omega$ are given in the Table 1. Meridional integration of this equation yields the expression for the initial temperature

$$
T(\phi, z)=\int^{\phi} \frac{\partial T\left(\phi^{\prime}, z\right)}{\partial \phi^{\prime}} d \phi^{\prime}+T_{0}(z) .
$$

The constant of integration $T_{0}(z)$ is chosen so that, at each level $z$, the global average of $T(\phi, z)$ is identical to $T_{\mathrm{US}}(z)$, the U.S. Standard Atmosphere, 1976 (COESA 1976) temperature. For easy reference, and to ensure reproducibility, we explicitly list in the appendix the numerical parameters that are needed to compute $T_{\mathrm{US}}(z)$. Finally, since the initial winds in (5) are identically zero at the ground, no surface geopotential needs to be specified to balance the initial condition.

The initial, unperturbed temperature profile resulting from (10) is shown in Fig. 1. To construct it, the derivative on the right-hand side of (9) is worked out analytically, and thus the integrand in (10) is evaluated exactly. Only the latitudinal integral needs to be performed numerically, but this can be done very accurately. We have used Gaussian quadrature, and have found that a mere 100 Gauss-Legendre points for the integral in (10) are sufficient to produce an initial balanced $T$ that is accurate to machine precision.
Because our initial zonal winds are very flat near the equator $\left[u(\phi) \propto \phi^{6}\right.$ for $\left.0<\phi \ll 1\right]$, as demonstrated by the nearly horizontal isentropes, the initial condition in Fig. 1 is stable with respect to symmetric instability. Furthermore, we have computed local Richardson numbers for our initial flow, and they are everywhere much larger than 1/4; this indicates that it is also stable to Kelvin-Helmholtz instabilities. Finally, we have checked that $N^{2}$ is everywhere positive, and thus the flow is stable to dry convective instability as well. Of course, our initial condition is baroclinically unstable: this is precisely what we are trying to set up.

In order to initiate the baroclinic instability, the zonal flow just described is perturbed by adding to the basic temperature profile a function $T^{\prime}$ in the form of a localized bump, centered in the midlatitudes and on the Greenwich meridian, specifically

$$
\begin{gathered}
T^{\prime}(\lambda, \phi)=\hat{T} \operatorname{sech}^{2}\left(\frac{\lambda-\lambda_{0}}{\alpha}\right) \operatorname{sech}^{2}\left(\frac{\phi-\phi_{0}}{\beta}\right) \\
\text { for }-\pi<\lambda<\pi,
\end{gathered}
$$

where $\lambda$ is longitude. Using an amplitude $\hat{T}=1 \mathrm{~K}$, this perturbation is applied identically at all pressure levels (the numerical values of the other parameters are given in Table 1). Note that we have not followed Thorncroft et al. (1993) who perturbed the flow with the most unstable linear mode; this procedure requires the cumbersome computation of the linear modes, which is surely something one would like to avoid unless necessary. Also, we have refrained from perturbing the flow with a single (or a couple) of zonal wavenumbers, such as $m=5$ or 6 ; while this is often done in the context of spectral models, it is not as natural for finite-difference or other spatial discretizations, and we have tried to avoid an initial condition that might bias or skew the solution in favor of a particular scheme.

The specification of the initial condition is completed by the choice of a uniform initial surface pressure $p_{s}=$ $p_{0}$.

\section{c. The need for explicit diffusion}

Having discussed the physical characteristics of the initial condition, we now return to discussing the need for the explicit diffusion terms in (1). It is well known that the initial conditions we have chosen lead to the development of sharp fronts over short periods of time (i.e., on the order of days). While we are aware of no formal proof, for all practical purposes the solution of the inviscid equations develop features that look like finite-time singularities. Therefore one cannot compute the solution of inviscid equations for very long given our initial condition.

It may be argued that the formation of fronts, inasmuch as it precludes the integration of inviscid equations, disqualifies the initial condition we have chosen. However, we contend that our choice of initial condition 
is, in fact, extremely relevant to climate modeling. First, baroclinic eddies are primary agents of temperature and momentum transport in the atmosphere; hence one would like to make sure that a given AGCM is able to compute them accurately. Second, the formation of steep horizontal gradients is one of the distinguishing features of high Reynolds number, nearly two-dimensional flows, such as large-scale atmospheric flows. Hence our choice of initial condition is highly realistic and of immediate practical interest for atmospheric modeling.

The choice of a simple diffusive operator to dissipate subgrid-scale processes is dictated, mainly, by the fact that it is easy to implement with most numerical schemes. In principle, other forms of diffusion could have been chosen, but most are specialized to specific schemes, or difficult to implement in general. For instance, $\nabla^{2 n}$ hyperdiffusion (with $n=2$ or $n=3$ ) acting on the vorticity/divergence equations is popular with spectral models, but it is very difficult to implement with finite-difference or spectral-element schemes for which the horizontal velocity is the prognostic variable. In section 5 we demonstrate how the choice of diffusion has a profound effect on the solutions. For the moment we simply caution the reader that, if the numerical model being tested does not solve the primitive equations with the diffusion terms exactly as specified in (1), it is unlikely that the computed solutions will reproduce the ones presented in this paper.

Before presenting the actual solutions, a word is needed as to the choice of the diffusion coefficient $\nu$. In atmospheric modeling studies, it is customary to choose the value of $\nu$ to be as small as possible for a given horizontal resolution, and to progressively decrease that value as the resolution is increased. This is, for instance, the procedure adopted by Boer and Denis (1997). In the present context, however, we are trying to demonstrate the convergence of our numerical solutions for a specific set of partial differential equations. If the value of $\nu$ were changed as the resolution was increased, we would effectively be changing the equations themselves, and hence would not expect convergence. In other words, if we were to decrease $\nu$ with increasing resolution, we would be trying to converge to the solution of the inviscid equations. As already argued, such solutions are likely to be singular, and thus not a good choice for a test case. Hence we need to keep the value of $\nu$ fixed as the resolution is increased. For the new test case, we have used the value $7.0 \times 10^{5} \mathrm{~m}^{2} \mathrm{~s}^{-1}$.

A final caveat: before computing the evolution of the flow, it is essential that the dynamical core be rid of all fudges (e.g., filters, fixers, smoothers, etc.) that are commonly found in AGCMs and not always explicitly documented. While these may be necessary for climate modeling, the inclusion of such procedures clearly amounts to solving different sets of equations than the ones specified here, and will thus likely lead to different results.

\section{Numerically converged solutions}

Having described how the new test case is to be set up, we now discuss how we have obtained numerically converged solutions. All the results in this section were computed using the Geophysical Fluid Dynamics Laboratory Flexible Modelling System (FMS) Spectral Dynamical Core (using the "Galway" release version), hereafter referred to as GFDL-SDC. In a nutshell, GFDL-SDC solves the primitive equations in $\sigma$ coordinates, using a pseudospectral transform method in the horizontal, a Simmons-Burridge finite-difference method in the vertical, and a Robert-Asselin filtered semiimplicit Crank-Nicholson/leapfrog scheme for time integration. These techniques are completely standard.

The evolution of the temperature field at the surface, ${ }^{1}$ for the first 12 days, ${ }^{2}$ is illustrated in Fig. 2. While this figure was constructed from a calculation using 20 vertical levels (equally spaced in $\sigma$ ), a horizontal trapezoidal truncation at wavenumber 341 (commonly denoted as a "T341L20") and a time step of $150 \mathrm{~s}$, we submit that it represents the numerically converged solution of (1), in the sense that this figure will not change (a) if the vertical, horizontal, or temporal discretizations are further refined, and (b) if it is computed with a different numerical scheme at sufficient resolution. We next demonstrate the first fact. The second one is discussed in the following section.

First, let us consider the horizontal resolution. In order to show that the solution in Fig. 2 is numerically converged, we plot in Fig. 3 a canonical quantity, the "eddy kinetic energy" (EKE) as a function of time, for five different horizontal resolutions: T21, T42, T85, T170, and T341, all with 20 vertical levels. The time steps used for these computations are 2400, 1200, 600, 300, and $150 \mathrm{~s}$, respectively. Following Thorncroft et al. (1993), EKE is defined as follows:

$$
\operatorname{EKE}(t)=\frac{1}{4 \pi a^{2}} \int \frac{1}{2}\left[(u-\bar{u})^{2}+(v-\bar{v})^{2}\right] \rho d V,
$$

where $\rho$ is the density, overbars denote zonal averages, and the volume integral is taken over the entire atmosphere. The fact that all the curves are basically superimposed might suggest that even a very low T21 resolution might be enough to compute accurate solutions. This, however, is not the case, as illustrated next.

\footnotetext{
${ }^{1}$ We use the term "surface" to indicate $\sigma=0.975$, which is very nearly the value of $\sigma$ at our lowest model level for a 20-level calculation. Since we are using a Simmons-Burridge (1981) scheme, the model levels $\sigma_{k}$ are located at values $\log \sigma_{k}=\left(\sigma_{k+1 / 2} \log \sigma_{k+1 / 2}-\right.$ $\left.\sigma_{k-1 / 2} \log \sigma_{k-1 / 2}\right) /\left(\sigma_{k+1 / 2}-\sigma_{k-1 / 2}\right)-1$, where $\sigma_{k+1 / 2}$ are the level interfaces. Thus, for a 20-level model, with levels of equal $\sigma$ thickness, the lowest model level is located at $\sigma=\exp [-0.95 \log (0.95) / .05-$ $1] \approx 0.974893147 .$. To avoid inflicting cruel and unusual punishment onto those wishing to reproduce our results, we extrapolate the fields onto $\sigma=0.975$ using a linear extrapolation.

${ }^{2}$ At the behest of a concerned reviewer of the original manuscript, we add that we are using here the solar definition of day (i.e., 1 day $=86400 \mathrm{~s}$ ) as opposed to the sidereal definition.
} 

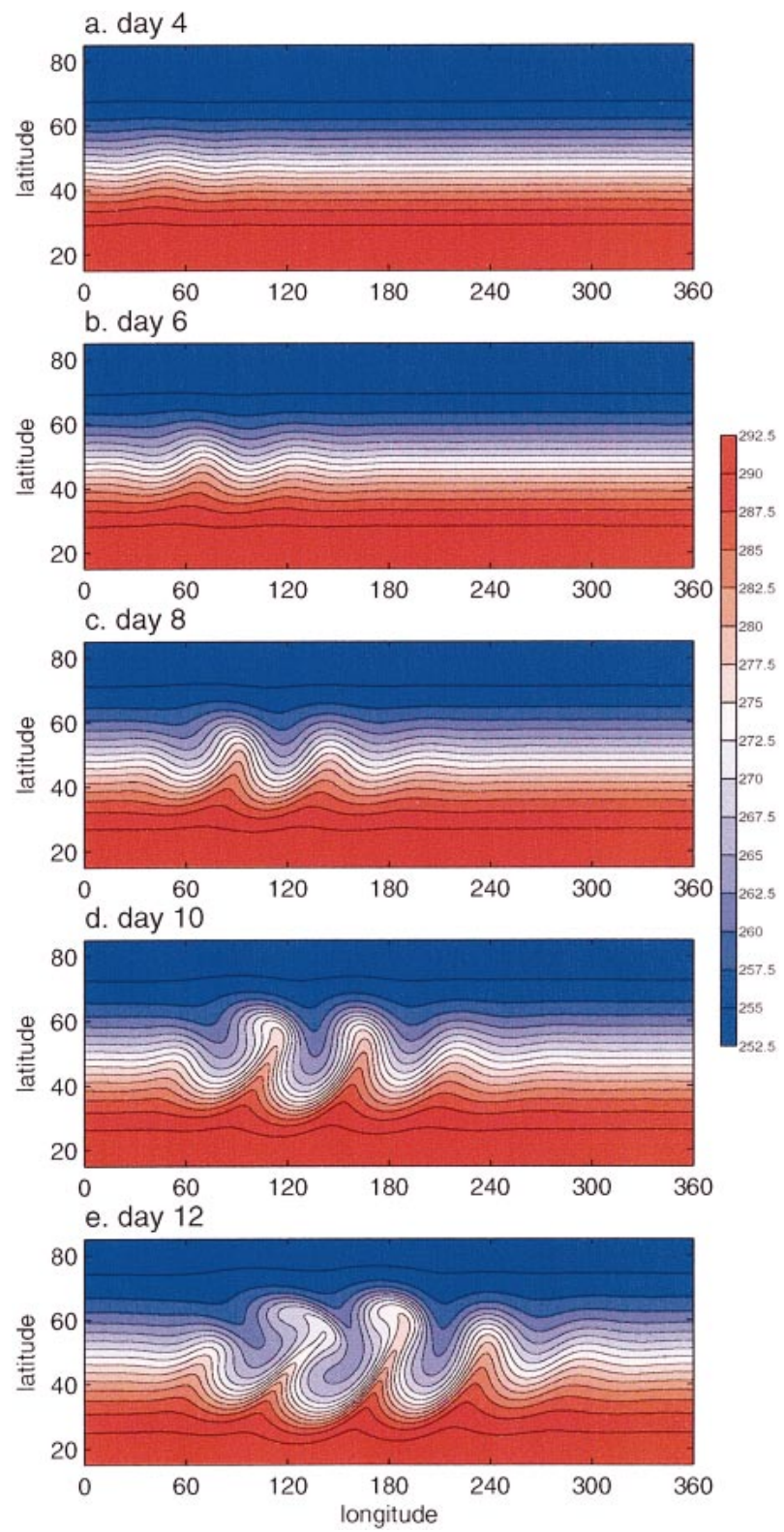

FIG. 2. The evolution from (a) day 4 to (e) day 12 of surface ( $\sigma$ $=0.975)$ temperature. The contour interval is $2.5 \mathrm{~K}$. This solution was computed with GFDL-SDC at T341L20 resolution.

In Fig. 4, the surface vorticity $\zeta$ at day 12 is plotted for the five resolutions from T21 to T341. At T42 $\zeta$ is still rather noisy, while at T85 it is nearly indistinguishable from the one at T170. One may thus consider the T85 solution as numerically converged for most-if not all-practical purposes. It should also be clear that there is nothing general about the fact that convergence is here achieved around T85. This value is only applicable to this test case and is a direct consequence of our choice of initial condition and of the value of the diffusivity $\nu$.

Finally, contrasting Figs. 4 and 3, one may correctly conclude that EKE is a highly inappropriate indicator

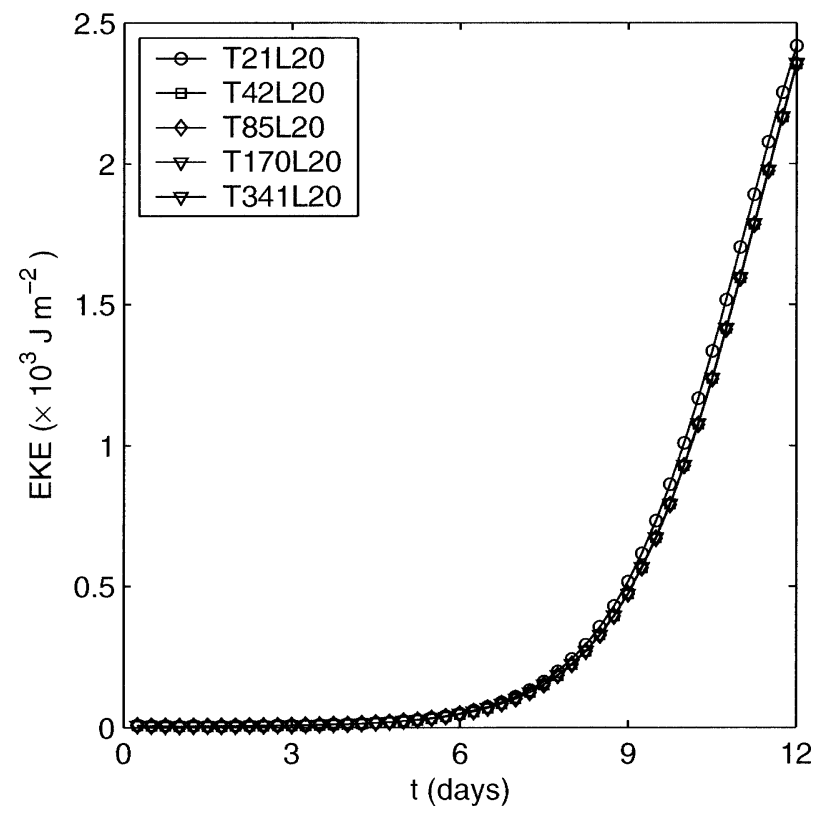

FIG. 3. The time evolution of EKE, as defined in (12), for five calculations of the GFDL-SDC, with 20 vertical levels and increasing horizontal resolution.

of numerical convergence. Ideally, one would like to construct error norms that are able to bring out differences that the eye is not able to detect from a contour plot. In the case of EKE, precisely the reverse occurs: the EKE suggests convergence while the contour plots tell the opposite story. This implies that more careful measures are needed to establish numerical convergence.

To this end we have computed several norms relating to the vorticity field. We wish to stress that $\zeta$ is not a differentiated quantity, but one of the prognostic variables in the GFDL-SDC [as well as in the Community Climate Model Version 3 (CCM3) and many other models]. It is the prognostic variables themselves that need to be looked at in order to establish numerical convergence, and not smoother integrated quantities (e.g., the geopotential) as is often done. Since both $T$ and $p_{s}$ are relatively smooth fields, we concentrate on $\zeta$ as a sensitive test of convergence (the divergence $\delta$ and the closely related vertical velocity $\omega$ are discussed below).

In Fig. 5a, we show the $L_{2}$ norm of $\zeta$ at the surface $(\sigma=0.975)$. This norm is defined as follows:

$$
\begin{aligned}
|\zeta|_{2}(\sigma, t) \equiv \frac{1}{\sqrt{4 \pi}} & \left\{\int_{-\pi / 2}^{\pi / 2} \cos \phi d \phi\right. \\
& \left.\times \int_{0}^{2 \pi} d \lambda[\zeta(\lambda, \phi, \sigma, t)]^{2}\right\}^{1 / 2}
\end{aligned}
$$

and has units of vorticity. Perhaps surprisingly, in view of Fig. 4, plots of $|\zeta|_{2}$ at the surface are qualitatively similar to those of EKE, in that curves for all five res- 

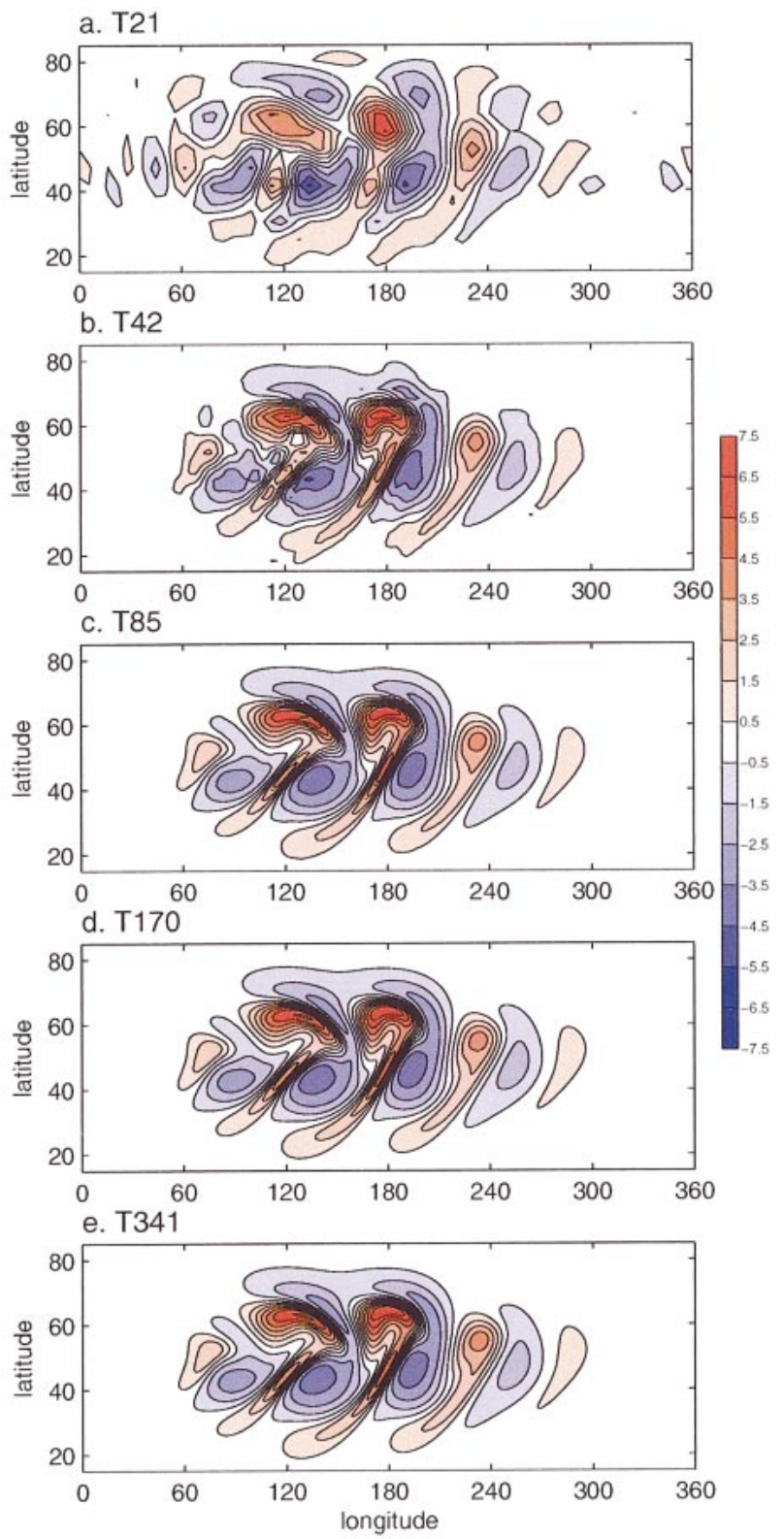

FIG. 4. The surface $(\sigma=0.975)$ vorticity, at day 12 , for five different horizontal resolutions ranging from (a) T21 to (e) T341. Contours from -7.5 to $7.5 \times 10^{-5} \mathrm{~s}^{-1}$, in steps of $1 \times 10^{-5} \mathrm{~s}^{-1}$; negative values are blue, and positive values are red. Results from GFDLSDC, with 20 vertical levels.

olutions are superimposed. Again, this suggests that the averaging involved in the computation of this norm masks some of the noisy features that are apparent in the actual plots of the vorticity field.

To apply a more stringent convergence test, we compute the $L_{\infty}$ norm of $\zeta$, defined by

$$
|\zeta|_{\infty}(\sigma, t) \equiv \max |\zeta(\lambda, \phi, \sigma, t)|,
$$

(the maximum is over all latitudes and longitudes) and plot its surface evolution in Fig. 5b. It is clear from this figure that the T21 and T42 calculations are noticeably different from their higher-resolution counterparts, as the contour plots in Fig. 4 attest.

To bring out even more differences among the lowand high-resolution calculations, it is necessary to compute higher derivatives. This is illustrated in Fig. 5c, where the $L_{\infty}$ norm of the gradient of $\zeta$ (more precisely of its magnitude) is plotted. Note that the T42 values are quite far from the converged values (even the T85 results are not completely identical to those at higher resolution). This is perhaps worrisome, given the dynamical importance of vorticity gradients for the propagation of Rossby waves (see, e.g., Scott et al. 2004). Computation of higher and higher derivatives, of course, will show differences at any resolution; hence we see little point in carrying this exercise further.

For readers who might be interested in more traditional measures of convergence, we offer in Fig. 6 the $l_{2}$ relative error norm of the differences between the lower resolutions and the T341 solution. Following Jakob-Chien et al. (1995), we define this as

$$
l_{2}(\zeta)=\frac{\left|\zeta-\zeta_{T}\right|_{2}}{\left|\zeta_{T}\right|_{2}}
$$

where the subscript $T$ indicates the T341 solution. In order to compute the numerator of $l_{2}(\zeta)$ with a maximum of accuracy, we first compute the Fourier/Legendre coefficients of $\zeta$, then pad the coefficients up to T341 with zeros, then transform back onto a T341 Gaussian grid, and finally compute the integral of the difference using Gaussian quadratures on the T341 grid. It should be clear from Fig. 6 that our numerical solutions are converging without problems, although the rate of convergence does not appear to be exponential.

In Fig. 7, a log-log plot of $l_{2}(\zeta)$ versus the number of degrees of freedom (DOFs) at day 12, it can be seen that the convergence rate with respect to the T341 solution is in fact algebraic. The dotted line has a slope of -0.955 . We suspect that the algebraic convergence is due to the nature of our initial conditions, that is, the fact that not all derivatives of $u$ as defined in (5) are continuous. A more careful choice of initial conditions would lead to exponential convergence. This, however, would change the problem definition significantly (and is left for future work). For the time being, we submit that Figs. 6 and 7 adequately demonstrate that our numerical solutions are converged with horizontal resolution.

Second, we address the question of vertical resolution. Surprisingly enough, convergence is more easily achieved in that case. In dealing with the vertical direction, it will prove useful to monitor a quantity that one might expect to be more sensitive to vertical discretization (rather than the vorticity $\zeta$ just discussed). We have chosen to use the vertical velocity $\omega$, defined in (3), as it is closely related to the divergence. Often 
a. $|\zeta|_{2}$

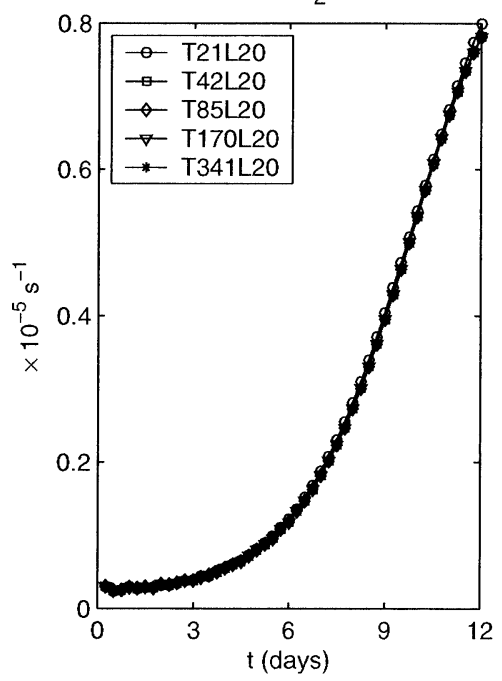

b. $|\zeta|_{\infty}$

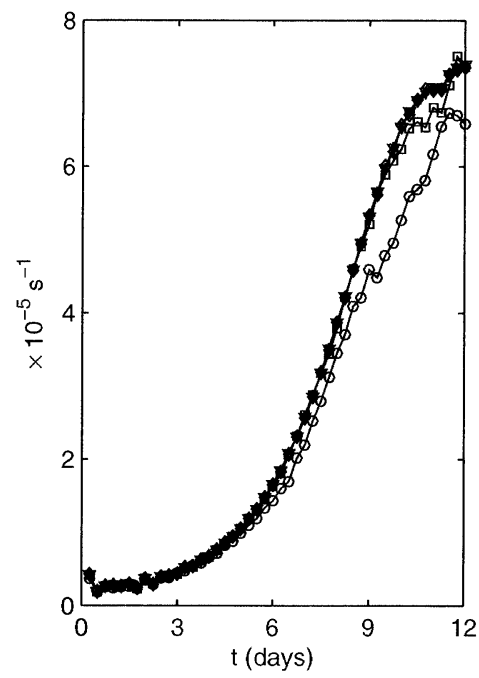

c. $|\nabla \zeta|$

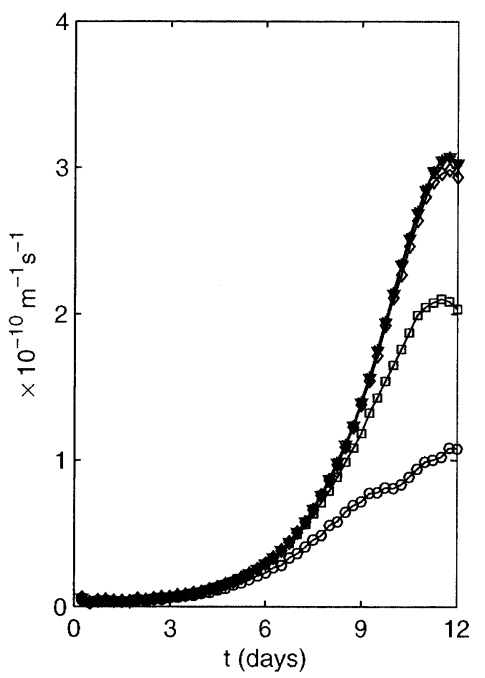

FIG. 5. Vorticity norms as a function of time, computed for the GFDL-SDC solution in Fig. 4. Each frame shows five calculations of increasing horizontal resolution, all with 20 vertical levels. The three norms are precisely defined in the text.

found to be rather noisy in AGCMs, the values of $\omega$ are crucially important to coupling the dynamical core to many physical processes, for example, convective parameterizations.

Using the horizontally converged resolution T85, we have performed five calculations with 10, 20 40, 80, and 160 levels, respectively. Noting that all the interesting action is concentrated in the midlatitudes, where the baroclinicity is originally strongest (cf. Fig. 2), we plot vertical cross sections of $\omega$ versus longitude at $\phi$

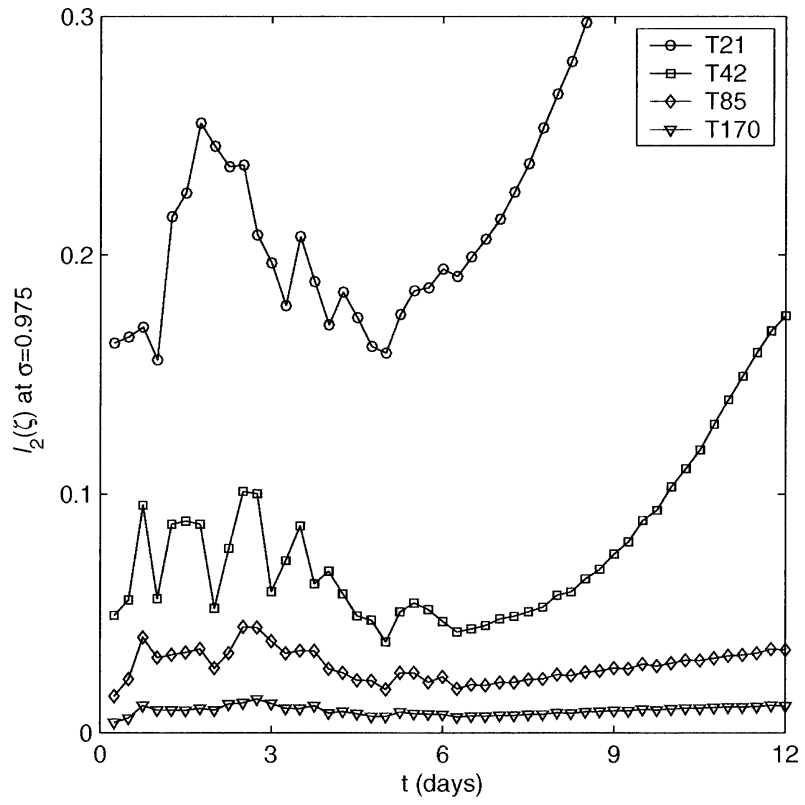

FIG. 6. Norm of the difference to the T341 solution, as defined in (15), computed with GFDL-SDC. All solutions, including the one at T341, are computed with 20 vertical levels. $=45^{\circ} \mathrm{N}$ and $t=12$ days. Results ${ }^{3}$ obtained using the GFDL-SDC are shown in Fig. 8 for the different number of levels.

An alternating sequence of updrafts and downdrafts

${ }^{3}$ As the Gaussian grid does not contain a point located at precisely $45^{\circ} \mathrm{N}$, we perform a linear interpolation in latitude to construct the cross sections of $\omega$ at $45^{\circ} \mathrm{N}$, shown in Fig. 8 .

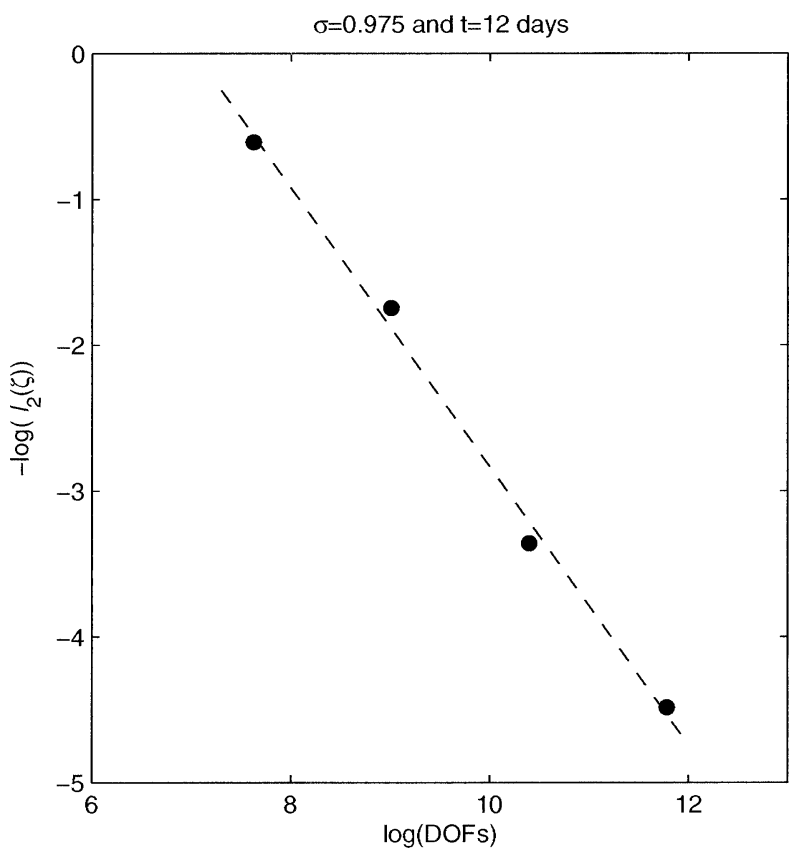

FIG. 7. Norm of the difference to the T341 solution, as defined in (15), at day 12, as a function of the DOFs. The four points correspond, from left to right, to the T21, T42, T85, and T170 solutions, respectively. The dashed line is a linear fit and has a slope of -0.955 . 

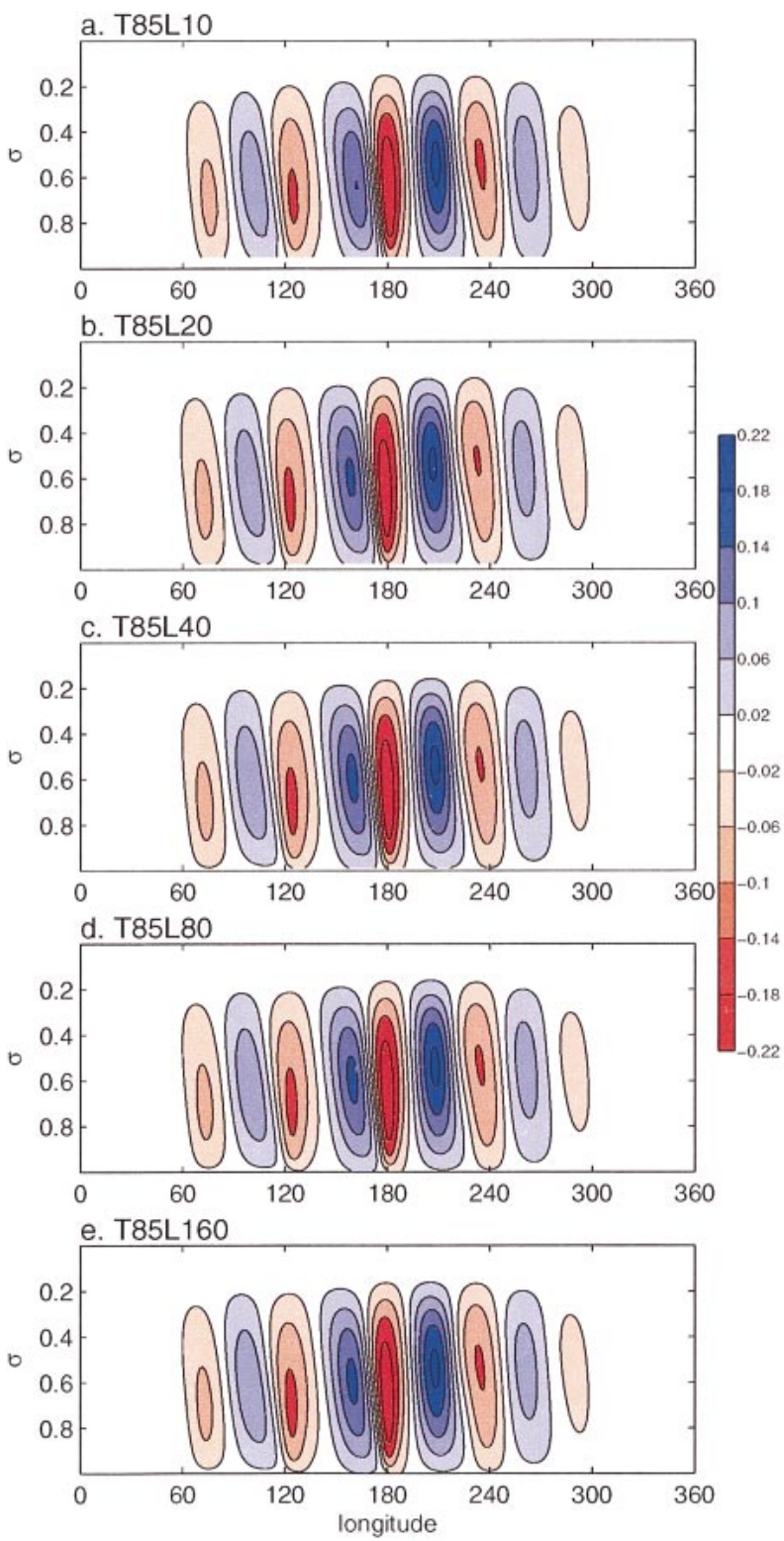

FIG. 8. Vertical cross sections of the vertical velocity $\omega$ at $\phi=$ $45^{\circ} \mathrm{N}$ and $t=12$ days, obtained with the GFDL-SDC at T85 resolution with (a) 10, (b) 20, (c) 40, (d) 80, and (e) 160 levels. Contours from -0.22 to 0.22 in steps of $0.04 \mathrm{~Pa} \mathrm{~s}^{-1}$. Red indicates upward motion, and blue indicates downward motion.

accompanies the positive and negative centers that develop in the vorticity field. Note that the features are extremely similar for all calculations, even near the surface. To further bring out the minuteness of the differences as the number of levels is increased from 10 to 160, we plot the values of $\omega$ at the specific height $\sigma=$ 0.5 in Fig. 9. For clarity, only the curves for 10 and 160 levels are shown, as the curves for 20,40, and 80 levels are contained between these two.

Third, having demonstrated the convergence of our numerical solution with vertical resolution, we finally

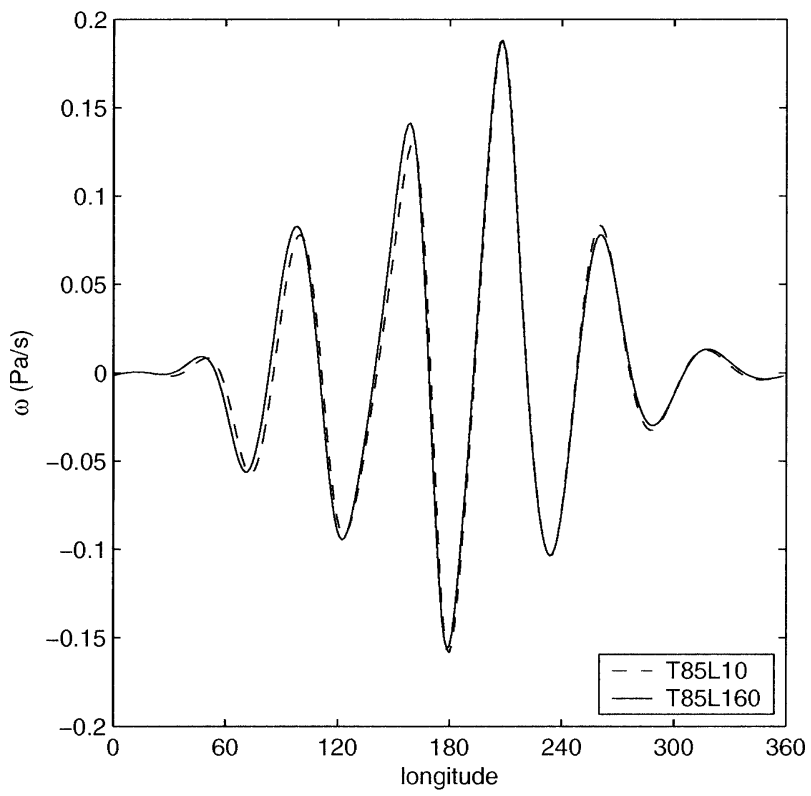

FIG. 9. The vertical velocity $\omega$, at $\sigma=0.5, \phi=45^{\circ} \mathrm{N}$, and $t=$ 12 days, obtained with the GFDL-SDC at T85. The solid line shows the values with 160 levels, the dashed line with 10 levels. The curves for 20,40, and 80 levels are contained between the two plotted curves and have been omitted for the sake of clarity.

address the question of temporal resolution. To evaluate this, we have computed T85L20 solutions with time steps of 600,300 , and $150 \mathrm{~s}$. Plots of the vorticity norms similar to those in Fig. 5 show that curves for these three different time steps are totally indistinguishable from each other. For this reason, we have avoided reproducing them here. With this last item, we believe to have convincingly demonstrated that our solutions, obtained with the GFDL-SDC, are numerically converged.

In summary then, solving the primitive equations as defined in (1), with the initial conditions spelled out in section 2, our semi-implicit pseudospectral model, with second-order finite-differences in the vertical, and a simple leapfrog time step, is able to obtain converged solutions with T85 horizontal resolution (roughly equivalent to a $1.4^{\circ}$ grid), 20 vertical levels, and a time step of $600 \mathrm{~s}$, for this particular value of $\nu$. These results can now be used as a point of comparison with other numerical schemes.

Finally, to assist modelers trying to reproduce this test case in the future, we offer in Table 2 a number of converged numerical values relating to the two key fields we have discussed, namely, the vorticity at the surface and pressure vertical velocity at $45^{\circ} \mathrm{N}$ after 12 days of integration. We suggest that future dynamical cores able to reproduce both the values in this table (to the two significant digits reported) and to visually match the converged surface $\zeta$ and $\omega$ at $45^{\circ} \mathrm{N}$ fields presented above should be considered to have "passed" the new test case. 
TABLE 2. Converged numerical values for the solutions to the test case described in section 3 and figures therein. All values are for $t=12$ days.

\begin{tabular}{lll}
\hline \hline \multicolumn{1}{c}{ Quantity } & \multicolumn{1}{c}{ Value } & Units \\
\hline EKE & $2.4 \times 10^{3}$ & $\mathrm{~J} \mathrm{~m}^{-2}$ \\
$|\zeta|_{2}$ at $\sigma=0.975$ & $7.8 \times 10^{-6}$ & $\mathrm{~s}^{-1}$ \\
$|\zeta|_{\infty}$ at $\sigma=0.975$ & $7.4 \times 10^{-5}$ & $\mathrm{~s}^{-1}$ \\
$|\nabla \zeta|_{\infty}$ at $\sigma=0.975$ & $3.0 \times 10^{-10}$ & $\mathrm{~m}^{-1} \mathrm{~s}^{-1}$ \\
$\operatorname{Max}(\omega)$ at $45^{\circ} \mathrm{N}$ & $1.9 \times 10^{-1}$ & $\mathrm{~Pa} \mathrm{~s}^{-1}$ \\
$\operatorname{Min}(\omega)$ at $45^{\circ} \mathrm{N}$ & $-1.7 \times 10^{-1}$ & $\mathrm{~Pa} \mathrm{~s}^{-1}$ \\
\hline
\end{tabular}

\section{Computational validation of the solutions}

Having demonstrated that one numerical scheme (in this case, the GFDL-SDC) is able to numerically converge as the spatial and temporal discretization is refined, we have at this stage no guarantee this converged solution is independent of the scheme we have used to obtain it. To the best of our knowledge, there is no rigorous general proof that solutions to the primitive equations exist or that they are unique. However, we are working under the assumption that all consistent numerical schemes will converge to an identical numerical solution.

To demonstrate that the converged numerical solution discussed above is not an artifact of the pseudospectral model that was used to compute it, we have adopted the practical expedient of computing a new set of numerical solutions to the identical set of equations and initial conditions but with a different numerical scheme. In what follows we show that the numerical solutions obtained with this other scheme converge to those obtained with the GFDL-SDC. Hence, for most practical purposes, we are able to claim that the numerical solutions we are presenting here ought to be reproducible by any numerical scheme, and that they establish a benchmark against which future dynamical cores can be tested.

To compute a new set of independent solutions to the equations and initial conditions of section 2, we use a dynamical core whose horizontal spatial discretization employs spectral elements in curvilinear coordinates on a cubed sphere, with second-order finite differences for the vertical discretization and advection. This dynamical core is known as the National Center for Atmospheric Research (NCAR) Spectral Element Dynamical Core (hereafter NCAR-SEDC) and is fully documented in Thomas and Loft (2004). We emphasize that the NCARSEDC not only is based on a completely different numerical method, but was compiled and executed on a different machine than the one used for the GFDL-SDC computations of the previous section.

In Fig. 10 we demonstrate the convergence of the NCAR-SEDC solution to the GFDL-SDC solution, by considering the surface $(\sigma=0.975)$ vorticity after 12 days of integration. The solid black contours show the NCAR-SEDC solution for five calculations with increasing horizontal resolution (and all with 20 equally
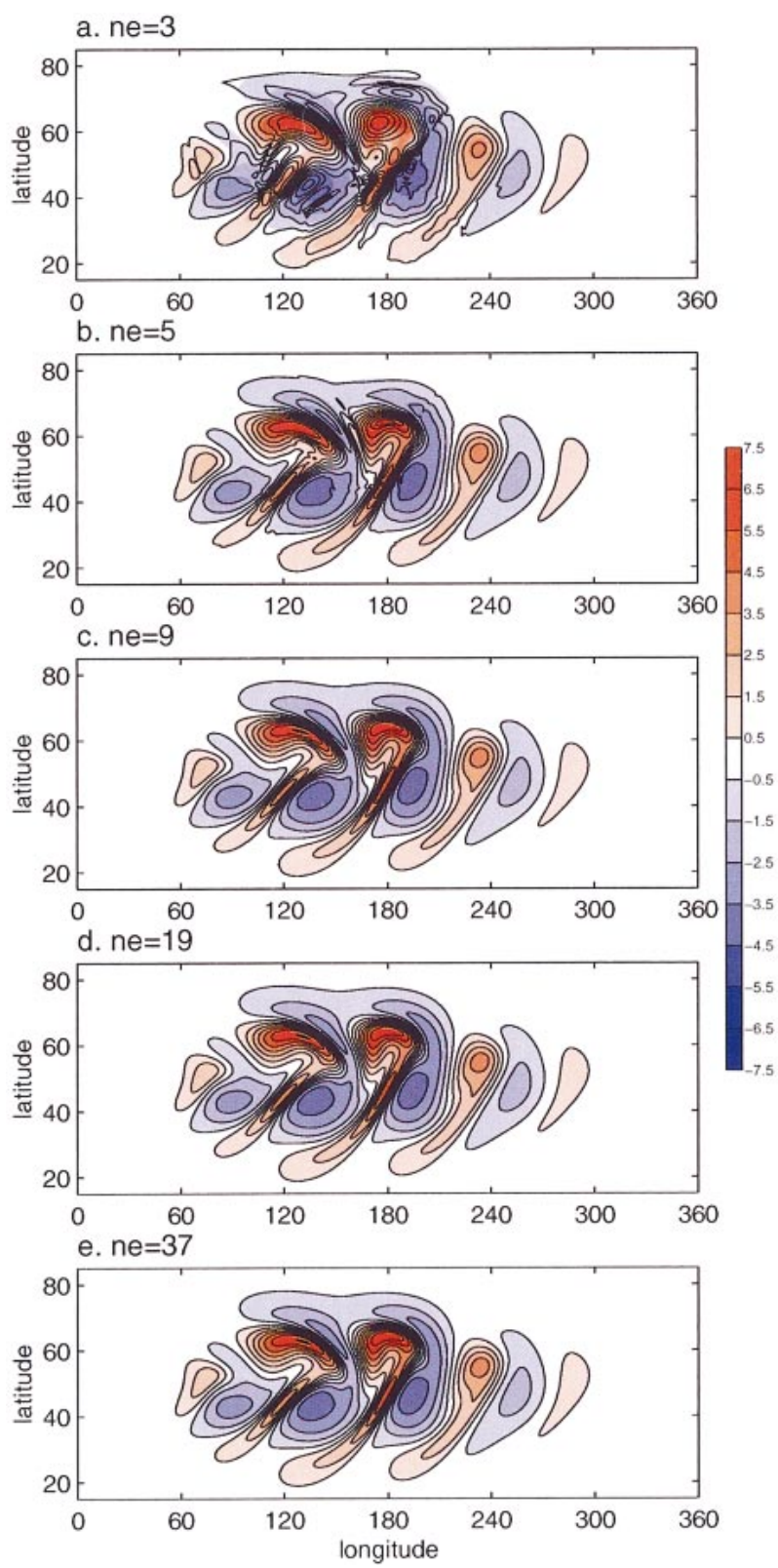

FIG. 10. Black contours: as in Fig. 4, but computed with NCARSEDC at increasing horizontal resolution. Contour levels as in Fig. 4. Colored patches: the GFDL-SDC solution at T341. All solutions computed with 20 vertical levels.

spaced vertical sigma levels). NCAR-SEDC computes the solutions using a cubed-sphere spectral-element method, for which the horizontal resolution is denoted by the parameter $n_{e}$, the number of spectral elements along one edge of one face of the cube. The total number of spectral elements in each NCAR-SEDC calculation is thus $6 n_{e}^{2}$. To compute the number of grid points used at a particular resolution, one multiplies this number by $64(8 \times 8)$, the number of Gauss-Lobatto-Legendre quadrature points in each element. Thus, for instance, an NCAR-SEDC integration at $n_{e}=37$ utilizes $64 \times$ 

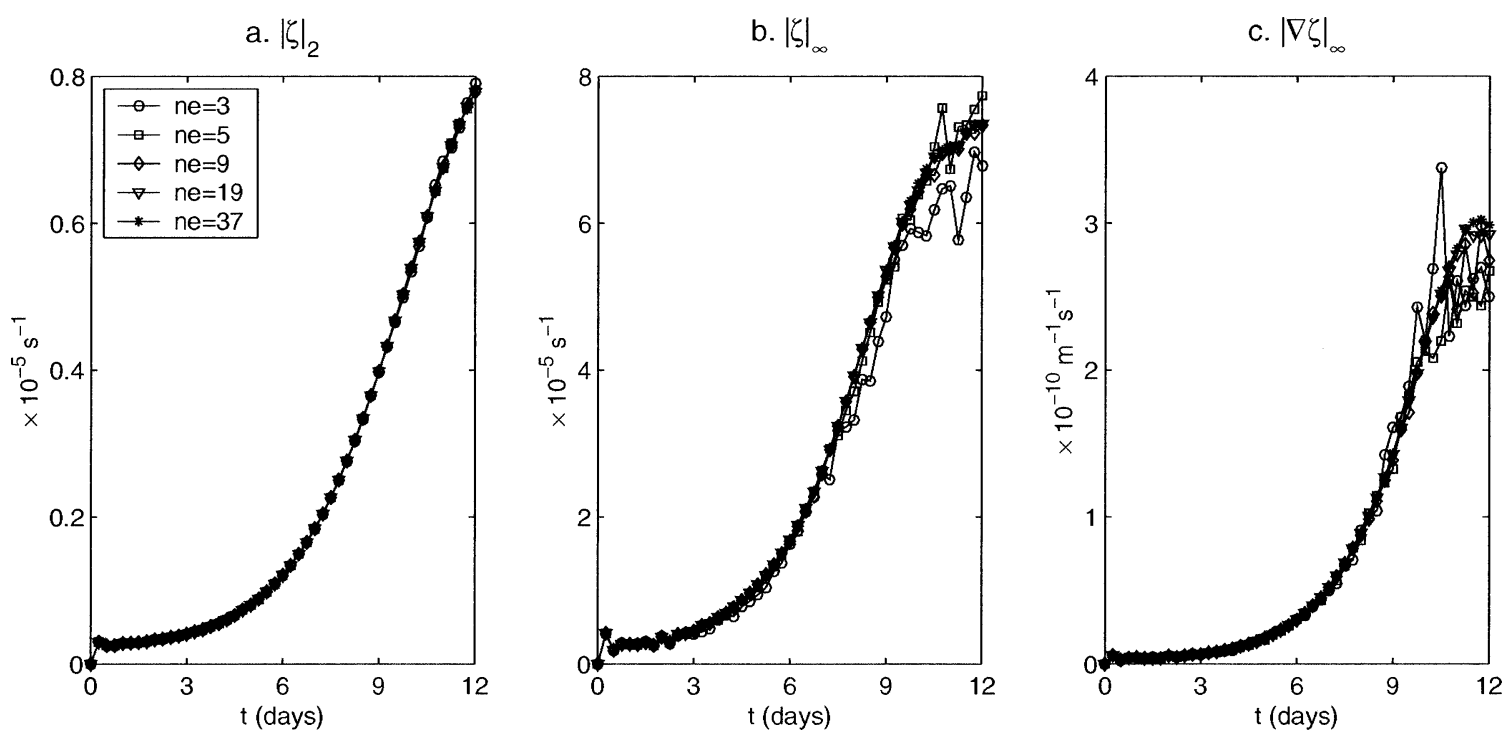

FIG. 11. As in Fig. 5, but for the NCAR-SEDC solutions illustrated in Fig. 10.

$6 \times 37^{2}=525696$ grid points; this is roughly equivalent to a GFDL-SDC integration at T341, which involves $1024 \times 512=524288$ Gauss-Legendre grid points. The horizontal resolutions for the five panels in Fig. 10 are chosen to be roughly equivalent to their pseudospectral counterparts in Fig. 4.

The key point in Fig. 10 is that, as the horizontal resolution is increased, the black contours of the NCARSEDC solutions become coincident with the color patches that represent the T341 GFDL-SDC solution. The agreement between colors and contours is already excellent with $n_{e}=5$, although some tiny differences can be detected. At $n_{e}=9$ the colors and contours are indistinguishably superimposed. Hence, as for the pseudospectral model, the spectral-element model appears to visually converge at a resolution comparable to T85.

In order to go beyond a merely visual demonstration, we plot in Fig. 11 the same norms for the surface vorticity field that were computed for the GFDL-SDC solutions. Although the manner in which convergence is achieved with each model is different (not exactly a surprising fact), the two models converge to the same numerical solution at sufficiently high resolution. In fact, the NCAR-SEDC curves at $n_{e}=37$ are completely superimposed on the GFDL-SDC ones at T341 (which we have omitted for clarity). Similarly, the numerical results in Table 2 are identical (to within the reported number of digits) for GFDL-SDC and NCAR-SEDC at the highest resolutions. We claim, therefore, that these numerical results represent the converged solution of system (1) with the initial conditions as specified.

Note that the numerical results reported in Table 2 are given to two significant figures. This is the number of digits to which the GFDL-SDC and the NCAR-SEDC solutions agree. As can be seen from Fig. 7, convergence for the pseudospectral GFDL-SDC is algebraic as op- posed to exponential, likely due to the fact that the initial velocity components are not infinitely differentiable. Thus, obtaining more digits would require much higher resolutions, well beyond the computational power available to us. In any event, the main objective of the present work is not to examine the convergence properties of a particular numerical method with respect to the initial condition, but to establish that one can compute a converged numerical solution to the primitive equations, independent of the numerical method.

For completeness, we offer in Fig. 12 the cross sections of $\omega$ at $45^{\circ} \mathrm{N}$ at day 12 computed with NCARSEDC, at different vertical resolutions (black contours) from 10 to 160 levels, and superimposed on the GFDLSDC solution (colored patches). Only at the lowest NCAR-SEDC vertical resolution is one able to detect the difference between the colors and the contours. Again, there should be little doubt that both models are converging to the same numerical solution.

\section{Effects of hyperdiffusion}

We offer in this section an example to demonstrate the importance of specifying exactly the same diffusion terms as in (1) if one is attempting to reproduce the numerically converged solutions presented above. While there is widespread practice of using a variety of methods to smooth out solutions in AGCMs, we wish to demonstrate here that indiscriminate use of such methods is likely to generate solutions that will differ substantially from the ones we have just presented.

With this goal in mind, we here compute a numerically converged solution to the dry, adiabatic, primitive equations with a different diffusion operator. Specifically, the terms on the right-hand side of (1) are replaced by the terms $-\nu \nabla^{4} \zeta,-\nu \nabla^{4} \delta$, and $-\nu \nabla^{4} T$ applied to the 

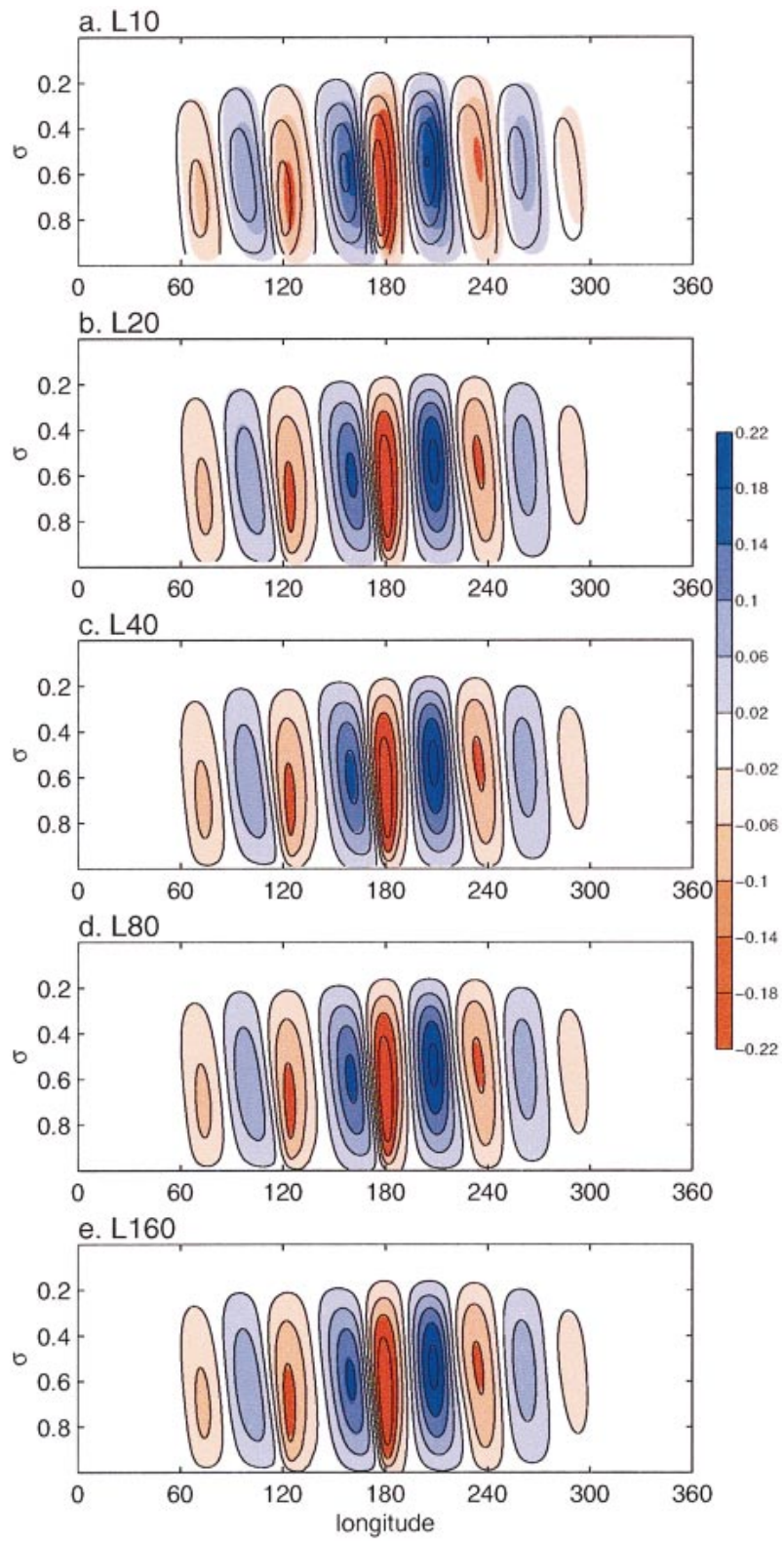

FIG. 12. Black contours: as in Fig. 8, but computed with NCARSEDC at $n_{e}=19$. Colored patches: the GFDL-SDC solution at a resolution of T85L160.

vorticity, divergence, and temperature equations, respectively (and not to the momentum equations themselves). Such a choice is very common with spectral methods and, in fact, is the one adopted by both NCAR's CCM3 and GFDL's Atmospheric Spectral Model (FMSAM2). Hence, consideration of $\nabla^{4}$ hyperdiffusion might be of interest in illuminating the kind of baroclinic instabilities that are computed by these well-established AGCMs.

For $\nu=2.5 \times 10^{16} \mathrm{~m}^{4} \mathrm{~s}^{-1}$, the numerical convergence of the vorticity field at 12 days is illustrated in Fig. 13. Note how different this solution is from the one with the regular diffusion operator in Fig. 4, especially in its ability to capture much steeper vorticity gradients. The contour interval in Fig. 13 is double the one in Fig. 4.

It is also interesting to note that, with this value of $\nu$, the T42 calculation is relatively noisy. Recall that the vorticity $\zeta$ is not a differentiated quantity; it is one of the prognostic variables. Thus the noise is due to the value of $\nu$ not being sufficiently large to produce smooth fields at T42. Perhaps surprisingly, both CCM3 and FMS-AM2, for which T42 is the standard horizontal resolution, use the even smaller value $\nu=1 \times 10^{16} \mathrm{~m}^{4}$ $\mathrm{s}^{-1}$ (Kiehl et al. 1996). The T42 solution, for that value, is even noisier and further from converged than the one shown here. These standard AGCMs, typically running at $\mathrm{T} 42$, are therefore computing rather noisy fields.

From Fig. 13 it appears that T170 is the visually converged resolution in this case. For completeness, we then show in Fig. 14 the cross-sectional vertical velocity at day 12, computed at T170 for 10 to 80 vertical levels (we were unable to fit a T170 calculation with 160 vertical levels on our machine). Again, note that the contour interval is double the one used in Fig. 8, indicating much stronger updrafts and downdrafts in this much less diffusive solution. It is perhaps not immediately obvious that one direct consequence of modifying the horizontal diffusion is to alter the vertical velocity magnitude by a factor of 2. This implies that physical parameterizations (e.g., convection schemes) as well as the vertical transport of constituents (e.g., water vapor) are likely to be profoundly impacted by the choice of horizontal diffusion in AGCMs.

Finally, while we do not expect the solutions with hyperdiffusion to be easily reproducible by models other than those based on the spectral method, we report a couple of values associated with the numerically converged solutions in Figs. 13 and 14. At 12 days, the maximum and minimum values of $\zeta$ at $\sigma=0.975$ are $15 \times 10^{-5}$ and $-8.4 \times 10^{-5} \mathrm{~s}^{-1}$, respectively, and the maximum and minimum values of $\omega$ at $45^{\circ} \mathrm{N}$ are 0.37 and $-0.32 \mathrm{~Pa} \mathrm{~s}^{-1}$.

\section{Summary and discussion}

We have computed and presented numerically converged solutions to the dry, adiabatic, primitive equations in spherical coordinates. The solutions were obtained with two completely independent numerical models. To the best of our knowledge, this is the first set of such solutions to have been computed. These solutions are meant to serve as benchmarks to help in the debugging and evaluation of future dynamical cores of AGCMs.

Specifically, we wish to propose a new test using the results presented here. It consists in three steps. First, starting from the initial conditions spelled out in section 2 , one would produce numerically converged instantaneous fields of $\zeta$ at the surface and $\omega$ at $45^{\circ} \mathrm{N}$ after 12 days of integration. At the risk of stating the obvious, 

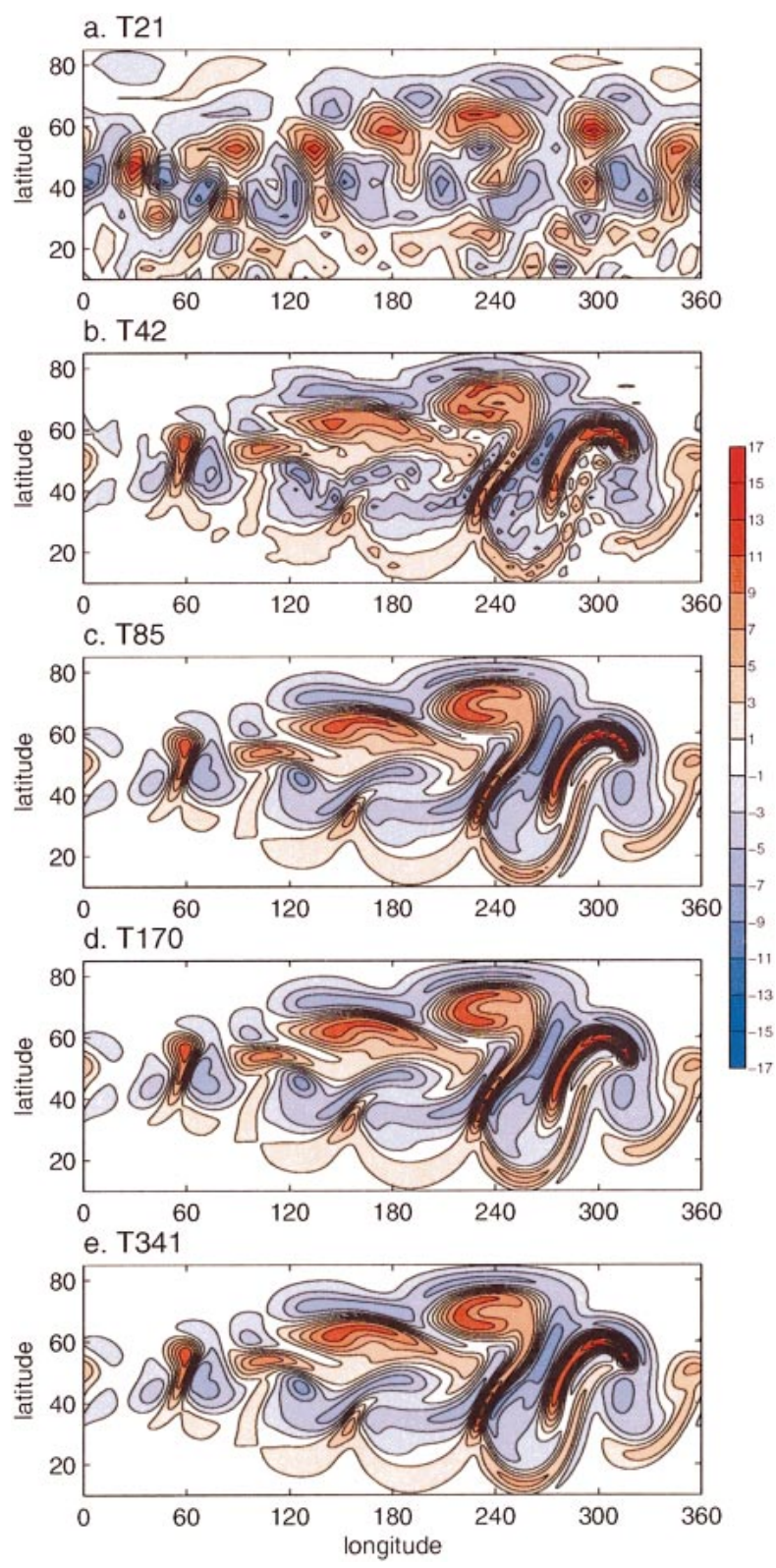

FIG. 13. As in Fig. 4, but for solutions with $\nabla^{4}$ hyperdiffusion. Contours from -17 to $17 \times 10^{-5} \mathrm{~s}^{-1}$, in steps of $2 \times 10^{-5} \mathrm{~s}^{-1}$.

we stress that there is little point in considering nonconverged numerical results since one is then unable to tell if the differences are due to truncation errors or coding errors.

The second step would consist of performing a visual comparison of $\zeta$ and $\omega$ with the fields presented above. The key qualitative features, number of positive and negative maxima, and their relative positions should visually match those presented in this paper. Finally, the third step would consist of comparing the values given in Table 2 with those computed with future dynamical cores. Agreement with the values in those tables should
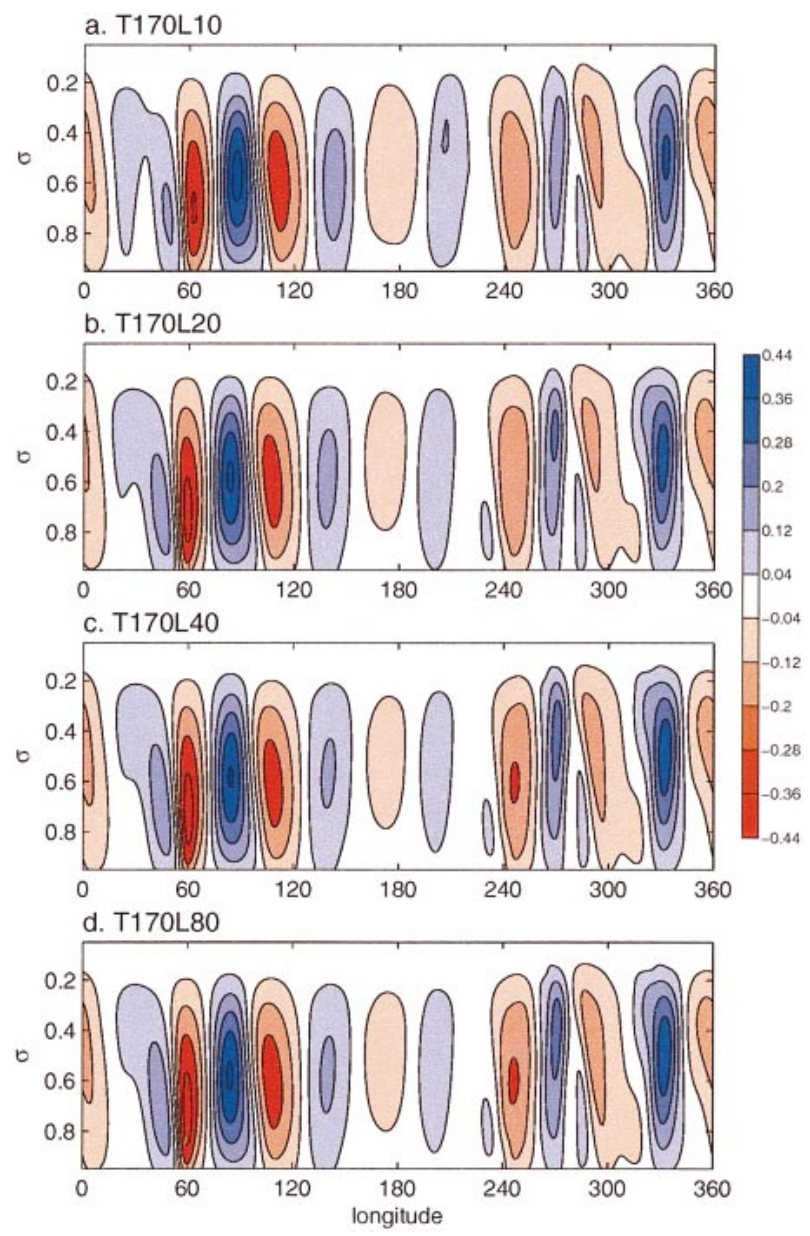

FIG. 14. As in Fig. 8, but for solutions with $\nabla^{4}$ hyperdiffusion (and excluding the 160 -level solution). Contours from -0.44 to $0.44 \mathrm{~Pa}$ $\mathrm{s}^{-1}$, in steps of $0.08 \mathrm{~Pa} \mathrm{~s}^{-1}$. Results from GFDL-SDC, with 20 vertical levels.

suffice to ensure that a model is free of serious coding errors or other deficiencies.

As pointed out in the introduction, we believe that the new test proposed here should serve a complementary role to the by-now classic HS94 benchmark. Once our test is passed, one may feel reasonably confident that a dynamical core is free from major bugs, and the next obvious step would then be to compute the statistics of the model with a 1000-day HS94 integration.

Finally, one might rightly ask whether our choice of including explicit diffusivity as part of the specifications of our new test is likely to limit its applicability. Specifically, the objection might be raised in the context of testing primitive equation models whose horizontal discretization does not require the explicit use of diffusivity (e.g., semi-Lagrangian schemes). Such schemes, typically constructed with conservation properties in mind, often involve implicit diffusion, and can thus be stably integrated without the need of explicit diffusivity. An example of current interest is the Lin-Rood (1996) 
scheme. One might argue that it is foolish to add explicit diffusion if a numerical scheme does not necessitate it.

Our answer to this objection is twofold. First, it should be clear that no obstacles exist to adding explicit diffusion to any scheme: to the degree that an identical set of partial differential equations is being solved, with identical initial and boundary conditions, and that $n u$ merically converged solutions are computed, any scheme should be able to reproduce the results presented in the paper. Second, if our initial conditions are integrated with no explicit diffusivity, it is highly unclear, in our opinion, what an implicitly diffusing scheme will converge to as the resolution is increased. As our initial condition is likely to generate infinite gradients in finite time in the absence of diffusivity, an implicitly diffusing scheme would presumably try to converge to a singular solution as the resolution is increased (and the implicit diffusion becomes negligible). Our suspicion, therefore, is that, for these initial conditions, numerical convergence may not be achievable with any scheme that does not contain explicit diffusion.

Acknowledgments. We gratefully acknowledge insightful conversations with Isaac Held, Paul Kushner, and Rich Loft. We also wish to thank Mr. Constantine Nenkov for a careful reading of the original manuscript. During the writing of this manuscript we have become aware of a somewhat similar test case being developed independently by Christiane Jablonowski and David Williamson; we wish to thank them for sharing their results with us prior to publication. This work is supported, in part, by an NSF grant to Columbia University, and by the generosity of the David and Lucile Packard Foundation. Last, and not least, we express our gratitude to Chief Editorial Assistant Mary Golden for her understanding and support.

\section{APPENDIX}

\section{The U.S. 1976 Standard Atmospheric Temperature Profile}

To compute the U.S. Standard Atmosphere, 1976 (COESA 1976) temperature, we use the canonical definition of $T_{\mathrm{US}}(z)$ as being piecewise linear in each of eight layers. The base $z_{i}$ of each layer and the lapse rate $\left(d T_{\mathrm{US}} / d z\right)_{i}$ in each layer are given in Table A1. From these, for $z_{i}<z<z_{i+1}$, the U.S. Standard Atmosphere, 1976 temperature is evaluated using the expression $T_{\mathrm{US}}(z)=T_{\mathrm{US}}\left(z_{i}\right)+\left(d T_{\mathrm{US}} / d z\right)_{i}\left(z-z_{i}\right)$. For $z=0$, we
TABLE A1. Numerical values of the bases $z_{i}$ and lapse rates $(d T /$ $d z)_{i}$ for the eight layers used to construct our piecewise linear expression for the U.S. Standard Atmosphere, 1976 temperature.

\begin{tabular}{cc}
\hline \hline$z_{i}(\mathrm{~km})$ & $(d T / d z)_{i}\left(\mathrm{~K} \mathrm{~km}^{-1}\right)$ \\
\hline 0 & -6.5 \\
11 & 0 \\
20 & +1.0 \\
32 & +2.8 \\
47 & 0 \\
51 & -2.8 \\
71 & -2.0 \\
80 & 0 \\
\hline
\end{tabular}

use $T_{\mathrm{US}}(0)=288.15 \mathrm{~K}$. Note that the formulas are being here applied to the $\log$ pressure height $z$, and not the "real" height.

\section{REFERENCES}

Boer, G. J., and B. Denis, 1997: Numerical convergence of the dynamics of a GCM. Climate Dyn., 13, 359-374.

COESA, 1976: U.S. Standard Atmosphere, 1976. U.S. Government Printing Office, $227 \mathrm{pp}$.

Durran, D. R., 1999: Numerical Methods for Wave Equations in Geophysical Fluid Dynamics. Springer-Verlag, 465 pp.

Galewsky, J., R. K. Scott, and L. M. Polvani, 2004: An initial-value problem for testing numerical models of the global shallow water equations. Tellus, in press.

Held, I. H., and M. J. Suarez, 1994: A proposal for the intercomparison of the dynamical cores of atmospheric general circulation models. Bull. Amer. Meteor. Soc., 75, 1825-1830.

Jakob-Chien, R., J. J. Hack, and D. L. Williamson, 1995: Spectral transform solutions to the shallow water test set. J. Comput. Phys., 119, 164-187.

Kiehl, J. T., and Coauthors, 1996: Description of the NCAR Community Climate Model (CCM3). NCAR Tech. Note NCAR/TN$420+$ STR.

Lin, S.-J., and R. B. Rood, 1996: Multidimensional flux-form semiLagrangian transport schemes. Mon. Wea. Rev., 124, 2046-2070.

Scott, R. K., D. G. Dritschel, L. M. Polvani, and D. W. Waugh, 2004 Enhancement of Rossby wave breaking by steep potential vorticity gradients in the winter stratosphere. J. Atmos. Sci., 61, 904-918.

Simmons, A. J., and D. M. Burridge, 1981: An energy and angularmomentum conserving vertical finite-difference scheme hybrid vertical coordinates. Mon. Wea. Rev., 109, 758-766.

Thomas, S. J., and R. D. Loft, 2004: The NCAR spectral element climate dynamical core: Semi-implicit Eulerian formulation. $J$. Sci. Comput., in press.

Thorncroft, C. D., B. J. Hoskins, and M. E. McIntyre, 1993: Two paradigms of baroclinic-wave life-cycle behavior. Quart. J. Roy. Meteor. Soc., 119, 17-55.

Williamson, D. L., J. B. Drake, J. J. Hack, R. Jakob, and P. N. Swarztrauber, 1992: A standard test set for numerical approximations to the shallow water equations in spherical geometry. J. Comput. Phys., 102, 211-224. 